\title{
On the Spherical Symmetry of a Static Perfect Fluid*
}

\author{
H. P. KÜNZLE \\ Department of Mathematics, University of California, Berkeley, California
}

Received September 28, 1970

\begin{abstract}
A globally static space-time with asymptotically Euclidean behavior representing a finite body of a perfect fluid and a vacuum region is shown to be diffeomorphic to Euclidean space and its metric spherically symmetric whenever the magnitude of the gravitational field strength is only a function of the gravitational potential. Under some additional physical assumptions it is then proved that this spherically symmetric solution is not deformable, that is, does not admit a nontrivial first order perturbation that is also a static, asymptotically Euclidean perfect fluid with the same equation of state and the same central value of the pressure and the gravitational potential.
\end{abstract}

\section{Introduction}

This paper deals with the equilibrium configurations of a nonrotating perfect fluid in a general relativistic framework. As in the Newtonian theory, where it was proved a long time ago (see Carleman [5] and for the more general context Lichtenstein [14]), this equilibrium was always believed to be attained for a spherically symmetrical configuration. The proof in the Newtonian theory used potential theory on three dimensional Euclidean space in a way that probably cannot be generalized to Riemannian manifolds.

Avez [1 and 2] suggested a very elegant argument based on Morse theory which, however, is only applicable in the very restricted case, where the gravitational potential $U$ has only nondegenerate critical points and the magnitude $W$ of the gravitational field strength, i.e. the length of the three-dimensional gradient of $U$, is a function of $U$ only. Under these assumptions it follows from the asymptotically Euclidean behavior that the 3 -space is in fact diffeomorphic to $\mathbb{R}^{3}$ and the space-time is spherically symmetric, i.e. admits $S O(3)$ as a isometry group with two-dimensional spacelike orbits.

The main result of this paper confirms the conjecture that the latter conclusion is probably independent of these restrictive hypotheses.

* This work was supported in part by the United States Atomic Energy Commission under contract number AT (04-3)-34, Project Agreement No. 125. 
It is shown that no first order static deviations from a spherically symmetric asymptotically Euclidean solution can exist, more precisely, that the spherically symmetric solution is not infinitesimally deformable in the space of all asymptotically Euclidean static perfect fluid spacetimes to a given equation of state, a given maximal (i.e. central) pressure $p_{0}$ and a minimal value $U_{0}$ of the gravitational potential $U$. This latter parameter is used to characterize the solution because of convenience. It could possibly be replaced by the total gravitational mass of the system.

The mathematics used in the proof is elementary and the argument could possibly be simplified if more were known about spaces of metrics on noncompact manifolds. Here this difficulty is partly overcome by using the fact that the equipotential surfaces are compact manifolds, indeed 2-spheres. In particular, while by the methods of Deser $[6,7]$ and Berger and Ebin [3] for distinguishing between isometric and essential deformations applied to the three-dimensional metric it is not very easy to identify a nondegenerate minimum of the energy for the spherically symmetric solution, this analysis is simplified when the equations are broken up into their restrictions to the equipotential surfaces and the $U$-dependence.

In the third section the result of Avez [1] is slightly generalized by dropping the assumption that the potential $U$ have no degenerate critical points. This would be useful if it could somehow be shown that $W=W(U)$ is true in general for an asymptotically Euclidean perfect fluid under certain physical assumptions - still a quite reasonable conjecture. If it could be done, it would follow that every static spacetime representing a (physically reasonable) perfect fluid that is asymptotically Euclidean is diffeomorphic to $\mathbb{R}^{4}$ and spherically symmetric.

Since it can be done at no additional cost we include the Newtonian case in the treatment - for comparison only since the methods of [5] and [14] are here considerably more effective.

\section{Static Perfect Fluids}

a) Static Space-Times

The definition is the one of Lichnerowicz [13], p. 109.

A space-time $M$ is called static iff there exists a 3-dimensional manifold $\Sigma$ and a diffeomorphism $\psi: M \rightarrow \Sigma \times \mathbb{R}$ such that

(i) $C_{x}=\psi^{-1}(\{x\} \times \mathbb{R})$ are timelike curves for all $x \in \Sigma$.

(ii) $\Sigma_{t}=\psi^{-1}(\Sigma \times\{t\})$ are (globally) spacelike hypersurfaces for all $t \in \mathbb{R}$.

(iii) $C_{x}$ for all $x \in \Sigma$ are tangent to a Killing vector field $\xi$ on $M$ that is orthogonal to all $\Sigma_{t}$. 
Then, if $M$ is geodesically complete, as we assume in the following, so is $\Sigma$ with the induced metric. We also assume that $\Sigma$ is orientable.

The metric on $M$ can be characterized by $g=\psi^{*}\left(-e^{2 U} d t^{2}+e^{-2 U} \gamma\right)$ where $\gamma$ is a complete Riemannian metric and $U$ a real valued function on $\Sigma$ and $d t^{2}$ the standard metric on $\mathbb{R}$. The function $U$ is called the gravitational scalar potential of the static field. Then $\psi^{*}\left(e^{2 U}\right)=g(\xi, \xi)$. (Cf. Ehlers and Kundt [8] for a general discussion of static fields in this formalism.)

As usual a static space-time $M$ (and the corresponding $(\Sigma, \gamma, U))$ is called spherically symmetric if $S O(3)$ is an isometry group of $(M, g)$ whose orbits are (generically) spacelike surfaces.

\section{b) Equations of a Static Perfect Fluid}

The Einstein equations for a perfect fluid with mass-energy density $\varrho$ and pressure $p$ in $M$,

$$
\stackrel{4}{R}_{\alpha \beta}-\frac{1}{2} \stackrel{4}{R} g_{\alpha \beta}=8 \pi\left[(\varrho+p) u_{\alpha} u_{\beta}+p g_{\alpha \beta}\right]^{1}
$$

assume in the static case the form

$$
\begin{aligned}
R_{i j} & =2 \partial_{i} U \partial_{j} U-2 \tilde{p} \gamma_{i j}, \\
\Delta U & =M
\end{aligned}
$$

where all geometric quantities refer to the 3-metric $\gamma, \Delta$ denotes the Laplace operator with respect to $\gamma$ and

$$
\tilde{p}=8 \pi p e^{-2 U}, \quad M=4 \pi(\varrho+3 p) e^{-2 U} .
$$

The Bianchi identities for the 3 -metric $\gamma$ are equivalent to the conditions

(i) the fluid satisfies an equation of state of the form $\varrho=\varrho(p)$,

(ii) pressure and density are functions of $U$ only, and are determined up to a constant by the equation of state by means of

$$
d p+(\varrho+p) d U=0,
$$

which can be written as

$$
\frac{d \tilde{p}}{d U}=-2 M
$$

Note that if (1) holds then (2), (3), and (4) are equivalent.

The equations of a perfect fluid subject to no other forces than its own gravitational attraction according to Newtonian hydrostatics are

$$
\Delta U=4 \pi \varrho \text { and } d p+\varrho d U=0
$$

${ }^{1}$ Conventions are as in Landau/Lifshitz [12], except that $\alpha, \beta, \ldots=0,1,2,3 ; i, j, \ldots$ $=1,2,3 ; A, B, \ldots=2,3 ; c=1, G=1$. 
where $\Sigma \simeq \mathbb{R}^{3}$ (diffeomorphic to $\mathbb{R}^{3}$ ) and $\gamma$ is the Euclidean metric on $\mathbb{R}^{3}$. If we treat the relativistic and the Newtonian case at the same time letting $v=0$ or 1 for Newtonian hydrostatics or general relativity, respectively, then

$$
\tilde{p}=8 \pi p e^{-2 v U} \text { and } M=4 \pi(\varrho+3 v p) e^{-2 v U},
$$

and the full set of equations is given by

$$
\begin{aligned}
& d p+(\varrho+v p) d U=0 \quad \text { or } \quad d \tilde{p} / d U=-2 M, \\
& \Delta U=M, \\
& R_{i j}=2 v\left(\partial_{i} U \partial_{j} U-\tilde{p} \gamma_{i j}\right) .
\end{aligned}
$$

\section{c) Physical Assumptions}

The essential condition in the Newtonian theory is $\varrho \geqq 0$ on $\Sigma$. This is already sufficient to prove the spherical symmetry of an equilibrium configuration if vacuum is assumed outside a compact domain and $\lim _{|x| \rightarrow \infty} U=0$, according to Lichtenstein [14].

In the relativistic case some limitation on the pressure is no doubt needed though a necessary condition is not known. (In Ref. [11] static space-times with positive mass density are constructed that have non Euclidean topology, but $p \leqq-\varrho$. They are not perfect fluids but could probably be made into such without a change of topology. See Bondi [4] for general limitations on $\varrho$ and $p$ following from the assumption that $\Sigma$ is topologically Euclidean and spherically symmetric.)

We assume for simplicity the certainly physically reasonable conditions

$$
0 \leqq 3 v p \leqq \varrho \quad \text { or equivalently } 0 \leqq 3 v \tilde{p} \leqq M
$$

and

$$
0<\frac{d p}{d \varrho}=\alpha^{2} \leqq \infty
$$

where $\alpha$ is usually interpreted as the velocity of sound in the fluid. Condition (9) implies for example that $M$ decreases outwards, for

$$
\frac{d M}{d U}=-4 \pi\left[\left(\alpha^{-2}+3 v\right)(\varrho+v p)+2 v(\varrho+3 v p)\right] e^{-2 v U}
$$

whence

$$
-\frac{d M}{d U}=4 \pi \alpha^{-2}(\varrho+v p) e^{-2 v U}+5 v M-3 v \tilde{p}
$$




\section{d) Asymptotically Euclidean Spaces}

It is essential that $\Sigma$ is topologically Euclidean near infinity. The conditions of asymptotical flatness used in gravitational radiation theory are therefore not (a priori) sufficient. We follow again Lichnerowicz [13] in defining $(\Sigma, \gamma, U)$ to be asymptotically Euclidean iff

(i) there exists a compact $K \subset \Sigma$ and a diffeomorphism $\varphi: \Sigma \backslash K \rightarrow \mathbb{R}^{3} \backslash B$ where $B$ is a closed ball centered at the origin,

(ii) with respect to the standard coordinate system in $\mathbb{R}^{3}$

$$
\begin{gathered}
\left.\gamma_{i j}-\delta_{i j}=0\left(|x|^{-1}\right), \quad \partial_{k} \gamma_{i j}=0(|x|)^{-2}\right), \\
U=0\left(|x|^{-1}\right), \partial_{k} U=0\left(|x|^{-2}\right) \quad \text { where } \quad|x|^{2}=\sum_{i=1}^{3}\left(x^{i}\right)^{2} .
\end{gathered}
$$

For simplicity we also assume that the matter distribution is finite, more precisely,

(iii) there exists a compact $Q \subset \Sigma$ such that $\varrho=p=0$ in $V=\Sigma \backslash Q$ and $M>0$ in the interior of $Q$.

As a consequence of these assumptions (i)-(iii), (6) and (8) we then have (cf. [13], p. 126 et seq.)

\section{Theorem 1.}

(a) $U<0$ on $\Sigma, \lim _{x \rightarrow \infty} U=0$ (i.e. for all $\varepsilon>0$ there exists a compact $K \subset \Sigma$ such that $|u|<\varepsilon$ in $\Sigma \backslash K)$,

(b) $\lim _{x \rightarrow \infty}|\nabla U|=0$,

(c) $U$ has no maximum and no minimum in the vacuum region $V$ and no critical point in some neighborhood of $\infty$ unless the space is flat,

(d) $D_{c}=\{x \in \Sigma / U(x) \leqq c\}$ is compact for all $c<0$,

(e) $S_{c}=\partial D_{c}=U^{-1}(\{c\}) \simeq S^{2}$ for $|c|$ sufficiently small.

By means of the classical expansion techniques it can be shown, moreover, that near infinity $U=-m /|x|+0\left(|x|^{-2}\right)$ and

$$
W=|\nabla U|=\left(\gamma^{i j} \partial_{i} U \partial_{J} U\right)^{1 / 2}=m /|x|^{2}+0\left(|x|^{-3}\right)
$$

where $m$ is the total (active gravitational) mass of the system defined by

$$
m=\frac{1}{4 \pi} \int_{D} M e^{3}=\frac{1}{4 \pi} \int_{i D}|\nabla U|^{2}
$$

where $D$ is any domain containing the support of $M, \stackrel{3}{e}$ the volume element of $\Sigma$ and ${ }^{2}$ the volume element to the induced metric on $\partial D$ (cf. [8], p. 68). 


\section{e) (2+1)-Dimensional Formulation of the Field Equations}

In any domain of $\Sigma$ where $U$ has no critical point the field equations can be written in terms of the induced metric $\bar{\gamma}$ of the equipotential surface $S_{c}$, its second fundamental form and the gradient square of $U$. Locally, in a 3-dimensional manifold we can even introduce an orthogonal coordinate system, but it will later be useful to have the complete formalism available (cf. Avez [1] and Sachs [17]). Let therefore $W^{2}=|\nabla U|^{2}$ $=\gamma^{i j} \hat{C}_{i} U \partial_{j} U$ and $n_{i}=W^{-1} \partial_{i} U$. Then, if in terms of a coordinate system $\left(x^{i}\right)=\left(U, x^{A}\right),(A=2,3)$

$$
d s^{2}=\gamma_{11} d U^{2}+2 P_{A} d x^{A} d U+\bar{\gamma}_{A B} d x^{A} d x^{B},
$$

where $\bar{\gamma}_{A B}$ is the induced metric of $S_{c}$, all capital indices are raised and lowered with respect to $\bar{\gamma}_{A B}$, quantities derived from $\bar{\gamma}_{A B}$ are barred, the second fundamental form of $S_{C}$ is defined by $\Omega_{A B}=\nabla_{A} n_{B}$ and ' $=\partial / \partial U$ then

$$
\bar{\gamma}_{A B}^{\prime}=2 W^{-1} \Omega_{A B}+2 \bar{\nabla}_{(A} P_{B)}
$$

and the field Eq. (7) translate into

$$
\begin{gathered}
\partial_{A} \Omega=\bar{\nabla}_{B} \Omega_{A}^{B}, \\
\bar{R}-\Omega^{2}+\Omega_{A B} \Omega^{A B}+2 v\left(W^{2}+\tilde{p}\right)=0, \\
W \Omega_{A B}^{\prime}+\Omega \Omega_{A B}-2 \Omega_{A}^{C} \Omega_{C B}-W^{-1} \bar{\nabla}_{A} \bar{\nabla}_{B} W+2 W^{-2} \partial_{A} W \partial_{B} W \\
-\left(2 v \tilde{p}+\frac{1}{2} \bar{R}\right) \bar{\gamma}_{A B} \\
-W\left(P^{C} \bar{\nabla}_{C} \Omega_{A B}+2 \bar{\nabla}_{(A} P^{C} \Omega_{B) C}\right)=0
\end{gathered}
$$

whence, by contraction with $\bar{\gamma}^{A B}$ and substitution from (14),

$$
\begin{aligned}
W \Omega^{\prime}-W^{-1} \bar{\Delta} W+2 W^{-2} \bar{\partial}^{A} W \partial_{A} W+\Omega_{A B} \Omega^{A B} \\
-2 v\left(W^{2}-\tilde{p}\right)-W P^{A} \partial_{A} \Omega=0 .
\end{aligned}
$$

Eq. (6) is equivalent to

$$
W^{\prime}=M W^{-1}-\Omega+P^{A} \partial_{A} W .
$$

\section{Spherically Symmetric Solutions}

a) Geometry of the Equipotential Surfaces when $W=W(U)$

In this whole section we assume that $W=|\nabla U|$ is a function of the potential $U$ only.

In any domain $G$ where $U$ has no critical point, using the coordinate system $\left(U, x^{A}\right)$, we have $\partial_{A} W=0$, thus by (2.17) $\Omega=\Omega(U)$, i.e. $\partial_{A} \Omega=0$. It then follows from (2.16) that $\Omega_{A B} \Omega^{A B}$ is a function of $U$ only. Finally 
(2.14) gives $R=R(U)$. Thus, $S_{c}$ is a surface of constant curvature for every regular value $c$ of $U$.

Lemma 1. If $M$ is a two-dimensional Riemannian manifold with metric $\bar{\gamma}_{A B}$ and $K_{A B}$ a symmetric tensor such that

$$
\text { (i) } \bar{\nabla}^{A} K_{A B}=0 \text { and (ii) } \bar{\gamma}^{A B} K_{A B}=K=\text { const. }
$$

then there exist a harmonic 1-form $\Phi$ on $M$ such that $K_{A B}=(1 / 2)\left(K-\bar{\Phi}^{2}\right) \bar{\gamma}_{A B}$ $+\Phi_{A} \Phi_{B}$ where $\bar{\Phi}^{2}=\bar{\gamma}^{A B} \Phi_{A} \Phi_{B}$.

Proof (Avez). There exists a function $f$ and a 1 -form $\Phi$ such that $K_{A B}=f \bar{\gamma}_{A B}+\Phi_{A} \Phi_{B}$. By (ii) $f=1 / 2\left(K-\widetilde{\Phi}^{2}\right)$. By (i) $\bar{\nabla}^{A} K_{A B}=2 \Phi^{A} \bar{\nabla}_{\mid A} \Phi_{B]}$ $+\left(\bar{\nabla}_{A} \Phi^{A}\right) \Phi_{B}=0$. Contracting with $\Phi^{B}$ gives $(\bar{\delta} \Phi) \Phi^{2}=0$. It follows that $\Phi$ is coclosed. Then $\Phi^{A} \bar{\nabla}_{[A} \Phi_{B]}=0$. But, locally, there exists a coordinate system such that $\Phi^{1}=0, \Phi^{2} \neq 0$, hence $\bar{\nabla}_{[2} \Phi_{1]}=0$, i.e. $\bar{d} \Phi=0$, thus $\Phi$ is closed.

In particular, if $M \simeq S^{2}$, according to the Hodge-de Rahm theory, there does not exist any harmonic 1 -form, thus $K_{A B}=1 / 2 K \bar{\gamma}_{A B}$ (see, for example, [10, p. 76]).

Applying this lemma to $\Omega_{A B}$ on $S_{c}$, when $S_{c}$ is a sphere (i.e. iff the curvature of $S_{c}$ is positive), we find $\Omega_{A B}=1 / 2 \Omega \gamma_{A B}$. Since locally we can assume that $P_{A}=0$, it follows from (2.12) that

$$
\bar{\gamma}_{A B}^{\prime}=W^{-1} \Omega \bar{\gamma}_{A B} .
$$

where $W^{-1} \Omega$ is a function of $U$ only. Therefore, if on some initial sphere $S_{c}$ the standard coordinate system $(\theta, \varphi)$ is used such that

$$
d \bar{s}^{2}=\bar{\gamma}_{A B} d x^{A} d x^{B}=r^{2}\left(d \theta^{2}+\sin ^{2} \theta d \varphi^{2}\right)
$$

where $r^{2}=2 / \bar{R}=r^{2}(U)$, then (1) can be integrated coordinatewise and shows that the form (2) of the metric remains valid for the neighboring surfaces $S_{c}$. Now $\Omega_{A B} \Omega^{A B}=1 / 2 \Omega^{2}$ and from (1) and (2)

$$
\Omega=2 W \frac{r^{\prime}}{r}=2 W \mathscr{R}^{\prime} \quad \text { where } \mathscr{R}=\ln r,
$$

so that the remaining nontrivial field equations become

and

$$
\left(W^{2}\right)^{\prime}+4 \mathscr{R}^{\prime} W^{2}=2 M
$$

$$
W^{2} \mathscr{R}^{\prime 2}=r^{-2}+v\left(W^{2}+\tilde{p}\right) .
$$

Recall now from Morse theory (Milnor [15], p. 14) that two surfaces $S_{c_{1}}$ and $S_{c_{2}}$ are diffeomorphic if there is no critical value of $U$ in $\left[c_{1}, c_{2}\right]$. 
From this. and the preceding remarks we get

Lemma 2. If all $c \in\left[c_{1}, c_{2}\right]$ are regular values of $U$ and any (whence all) $S_{c}$ is a sphere then $D_{c_{1} c_{2}}=U^{-1}\left[\left(c_{1}, c_{2}\right)\right]$ is diffeomorphic to $S^{2} \times \mathbb{R}$ and the metric in $D_{c_{1} c_{2}}$ is given by

$$
d s^{2}=W^{-2} d U^{2}+r^{2}\left(d \theta^{2}+\sin ^{2} \theta d \varphi^{2}\right)
$$

where $r(U)$ and $W(U)$ are determined up to constants from (4) and (5). In other words, $\left(D_{c_{1} c_{2}}, \gamma, U\right)$ is spherically symmetric.

\section{b) Asymptotic Behavior of $U$}

From Theorem 1 and Lemma 2 it follows that $S_{c} \simeq S^{2}$ for all $c>c_{*}$ where $c_{*}$ is the greatest critical value of $U$. If moreover $U$ is assumed to be a Morse function (i.e. has only isolated critical points and not two with the same value) then the method ov Avez [1] gives the result immediately. We do not need this assumption, however, if we proceed directly.

Integration of (4) gives

$$
W=\frac{m}{r^{2}}
$$

where $m^{2}(U)=2 \int_{U_{0}}^{U} M r^{4} d U$ for some $U_{0}$. Since in the vacuum region $m(\geqq 0)$ is a constant, in fact equal to the total mass (2.11) of the system, we assume that $m>0$, since otherwise $W=0$ would imply a flat vacuum region.

For the vacuum region (5) can be explicitly integrated leading to the exterior Schwarzschild (or Newtonian) potential in our coordinates. In particular, since $W$ never vanishes $U$ has no critical points in the vacuum region.

\section{c) Behavior of $U$ Near a Critical Point}

First observe that, since we assume $W=W(U)$ to hold on all of $\Sigma$, either $S_{c}=U^{-1}(\{c\})$ is a regular submanifold (for regular values $c$ ) or $S_{c}$ is contained in the critical set of $U$. Thus it is enough to consider all critical values of $U$.

Let now $x_{0}$ be a critical point of $U$. From $W=W(U)$ and $\partial_{i} U\left(x_{0}\right)=0$ it is easy to deduce

$$
\left(W^{2}\right)^{\prime} U_{i j}=2 U_{i r} U_{j}^{r}
$$


where $U_{i j}=\nabla_{,} \nabla_{i} U\left(x_{0}\right)$. With respect to an orthonormal frame at $x_{0}$ the only possibilities thus are

$$
\left.\begin{array}{lll}
\text { (I) } \quad U_{i j}=\frac{1}{3} M_{0} \delta_{i j}, & & \\
\text { (II) } \quad\left(U_{i j}\right)=\frac{1}{2} M_{0}\left(\begin{array}{lll}
1 & & \\
& 1 & \\
& & 0
\end{array}\right),\left(W^{2}\right)^{\prime}=\frac{2}{3} M_{0}, \\
\text { III) } \quad\left(U_{i j}\right)=M_{0}, \\
& \left.\begin{array}{lll}
1 & & \\
& 0 & \\
& & 0
\end{array}\right),\left(W^{2}\right)^{\prime}=2 M_{0} .
\end{array}\right\}
$$

(Note that $M_{0}>0$, since $U$ has no critical point in vacuum.) In each case $\left(W^{2}\right)^{\prime}>0$, thus a critical value is always isolated.

To study the implications of the other equations near a critical point we introduce a normal coordinate system centered at $x_{0}$ and adapted to a frame of eigenvectors of $U_{i j}$. Then

$$
\begin{aligned}
& U-U_{0}=\frac{1}{6} M_{0}\left(y^{1^{2}}+y^{2^{2}}+y^{3^{2}}\right)+0\left(|y|^{3}\right), \\
& U-U_{0}=\frac{1}{4} M_{0}\left(y^{1^{2}}+y^{2^{2}}+0\left(|y|^{3}\right),\right. \\
& U-U_{0}=\frac{1}{2} M_{0} y^{1^{2}}+0\left(|y|^{3}\right)
\end{aligned}
$$

in case I, II and III, respectively. Thus, the function $z=\gamma^{-1 / 2}\left(U-U_{0}\right)$ where $\gamma=\frac{1}{6} M_{0}, \frac{1}{4} M_{0}$ or $\frac{1}{2} M_{0}$ in case I, II or III behaves exactly like a radial polar, radial cylindrical or cartesian coordinate, respectively, in the Euclidean tangent space to $x_{0}$ in the limit for $|y| \rightarrow 0$. The functions $W, r, M, \tilde{p}$ depend only on $z$ and it is easy to discuss their limits when $z$ approaches zero.

Eqs. (4) and (5) become in terms of $z$,

and

$$
r \partial_{z} W^{2}+4 W^{2} \partial_{z} r=4 \gamma z M r
$$

$$
W^{2}\left(\partial_{z} r\right)^{2}=4 \gamma^{2} z^{2}\left[1+r^{2}\left(W^{2}+\tilde{p}\right)\right] .
$$

Now by (8) $W_{0}=0$ and $\left(W_{0}^{2}\right)^{\prime}=4 \gamma$, whence $W^{2}=4 \gamma^{2} z^{2}+0\left(z^{3}\right)$. Due to its geometrical meaning $r$ must be a regular function of $z, r=r_{0}+r_{1} z+0\left(z^{2}\right)$, say. Similarly, $M$ and $\tilde{p}$ must be regular functions of $U$ near $x_{0}$ (recall that $M_{0}^{\prime}$ is related to the velocity of sound), $M=M_{0}+\gamma M_{0}^{\prime} z^{2}+0\left(z^{4}\right)$ and by (2.5) $p=p_{0}-2 \gamma M_{0} z^{2}+0\left(z^{4}\right)$.

Comparison of the coefficients of the first powers of $z$ shows that necessarily case I occurs iff $r_{0}=0$ and case III iff $r_{0} \neq 0$. All functions are then completely determined by (9) and (10).

Now suppose $U_{0}=c_{*}$, the greatest critical value of $U$. Then if $x_{0} \in S_{c_{*}}$ is of type III $S_{c_{*}}$ must be a sphere of radius $r_{0}$ as only possible limit of the 
spheres $S_{c}$ for $c \downarrow c_{*}$ or $z \downarrow 0$. But since $U=U_{0}+1 / 2 M_{0} z^{2}$ in this case $U$ would have to increase again for negative values of $z$, which would contradict the fact that all $S_{\mathrm{c}} \simeq S^{2}$ for $c>c_{*}$. It follows that $x_{0}$ must be of type I, that is an isolated nondegenerate minimum of $U$. Then, however, $U$ increases in all directions from $x_{0}$ so that $x_{0}$ is in fact the only critical point of $U$ and moreover, $\Sigma \simeq \mathbb{R}^{3}$ (cf. Morse [16]).

We collect these results in

Theorem 2. If in a static space-time $M$ representing a compact body of a perfect fluid with $\varrho+3 v p \geqq 0$ and an asymptocically Euclidean vacuum region the gravitational field strength $W$ is a function of the potential $U$ then $M$ is diffeomorphic to $\mathbb{R}^{4}$ and spherically symmetric.

Note that it follows from (4) and (5) that $r^{\prime}>0$ everywhere, so that both $r$ and $U$ are functions monotonically strictly increasing from the center outwards. If the equation of state $\varrho=\varrho(p)$ and the central value of, for example, the pressure, $p_{0}$, and $U_{0}$ are given $p, M, r$ and $W$ are uniquely determined by (2.3), (2.4), (4), (5) and their initial values given in the last subsection. The value $U_{s}$ of $U$ at the surface of the body is the solution of $p(U)=0$ and the total mass can be obtained from $m_{s}=W\left(U_{s}\right) r^{2}\left(U_{s}\right)$. Similarly, prescribing $\varrho(p), m_{s}$ and $r_{s}$ determines everything.

\section{Static Deformations of a Spherically Symmetric Solution}

\section{a) Isometric Deformations}

Let $\mathscr{S}$ be the set $\{(\gamma, U)\}$ of asymptotically Euclidean pairs of space metrics and potentials on a given $\Sigma \simeq \mathbb{R}^{3}$ that satisfy the equations of a perfect fluid with a given equation of state $\varrho(p)$ and a fixed $U_{0}=\min (U / x \in \Sigma)$ and $p_{0}=p\left(U_{0}\right)$. According to the last section there is a unique $(\gamma, \boldsymbol{U}) \in \mathscr{S}$ (up to isometry) that is spherically symmetric. In any case, $\tilde{p}(U)$ and $M(U)$ are uniquely determined, hence also $U_{s}$ as solution of $\tilde{p}(U)=0$.

Since in the Newtonian case (where of course $\gamma$ is fixed) it is known that there is only one element of $\mathscr{S}$, namely the spherically symmetric one, it is reasonable to expect that in the relativistic case there should be only one equivalence class (with respect to isometry) of $(\gamma, U)$ in $\mathscr{S}$. The purpose of this section is to prove this in the infinitesimal version namely that there are no first order deformations of $(\gamma, \boldsymbol{U})$ which do not arise merely from coordinate changes.

Remark. This program can also be phrased as follows. Let

$$
\begin{aligned}
\psi: \operatorname{Diff}(\Sigma) \times \mathscr{S} & \rightarrow \mathscr{S} \\
(a,(\gamma, U)) & \mapsto \psi_{a}[(\gamma, U)]=\left(\psi_{a}^{*}, \cdot \psi_{a}^{*} U\right)
\end{aligned}
$$


be the action of the group of diffeomorphisms of $\Sigma$ on $\mathscr{S}$. If

$$
\begin{aligned}
\psi_{(\gamma, u)}: \operatorname{Diff}(\Sigma) & \rightarrow \mathscr{S} \\
a & \mapsto \psi[a,(\gamma, U)]
\end{aligned}
$$

then $\psi_{(\gamma, u)}[\operatorname{Diff}(\Sigma)]$ is the orbit of $(\gamma, U)$ under $\operatorname{Diff}(\Sigma)$. Then, if the tangent space to the orbit through $(\gamma, U)$ coincides with the whole tangent space at $(\gamma, U)$, i.e. if $T_{e} \psi_{(\gamma, U)}: T_{e}(\operatorname{Diff}(\Sigma)) \rightarrow T_{(\gamma, U)} \mathscr{S}(e=$ identity in $\operatorname{Diff}(\Sigma))$ is surjective, $(\gamma, U)$ is called infinitesimally stable under $\operatorname{Diff}(\Sigma)$. In this terminology we propose to prove that the spherically symmetric $(\gamma, U)$ in $\mathscr{S}$ is infinitesimally stable under $\operatorname{Diff}(\Sigma)$.

Let again $(\gamma, \boldsymbol{U})$ be the spherically symmetric solution in $\mathscr{S}$. Since $U$ has only one nondegenerate critical point the same must be true for all $U$ in a sufficiently small $C^{2}$-neighborhood $\mathscr{V}$ of $(\gamma, U)$ in $\mathscr{S}$. Therefore any $(\gamma, U) \in \mathscr{V}$ can also be described in terms of $\bar{\gamma}$ and $W$ as functions of $U$ and, possibly, angular variables on $S_{c}$. All $S_{c}$ for $c \neq U_{0}$ will still be diffeomorphic to $S^{2}$, though no longer necessarily of constant curvature.

It follows that the tangent space to $(\gamma, \boldsymbol{U})$ can be described in terms of a symmetric tensor field $h$ and a function $\omega$ on $S_{c}$ for all values $c$ of $U$, more precisely, the first order deformations can be given by

and

$$
\bar{\gamma}_{A B}=\bar{\gamma}_{A B}+\lambda h_{A B}
$$

$$
W=W(1+i \omega)
$$

where $\lambda$ is the parameter of the curve in $\mathscr{S}$ tangent to $(h, \omega)$ in $(\gamma, \boldsymbol{U})$. For a fixed value $U=c$ it is known that the symmetric tensor $h$ on the compact Riemannian manifold $S_{c}$ can be uniquely decomposed into a symmetric tensor with vanishing divergence and a Killing derivative, i.e.

where

$$
h_{A B}=\Phi_{A B}+2 \nabla_{(A} \xi_{B)}
$$

$$
\widetilde{\nabla}_{A} \Phi_{A B}=0
$$

and the vector field $\xi$ on $S_{c}$ is the Killing field of a 1-parameter group of diffeomorphisms of $S_{c}$ (cf. Berger and Ebin [3]).

We now consider the 3-metric $\gamma$ as a family of 2-metrics $\bar{\gamma}$, of functions $W$ and vector fields $P$ on $S_{c}$, parametrized by $U$, namely such that $d s^{2}=\left(W^{-2}+P_{C} P^{C}\right) d U^{2}+2 P_{A} d x^{A} d U+\bar{\gamma}_{A B} d x^{A} d x^{B}$. A $(\gamma, U)$ is then in the orbit under $\operatorname{Diff}(\Sigma)$ of $(\gamma, \boldsymbol{U})$ if the corresponding $(\bar{\gamma}, W, P)$ can be obtained from $(\bar{\gamma}, \boldsymbol{W}, 0)$ by a diffeomorphism $\varphi$ of the form

$$
x^{A}[\varphi(x)]=\varphi^{A}\left(x^{B}, U\right), \quad U[\varphi(x)]=U(x) .
$$

Infinitesimally, for $\bar{\gamma}$ and $W$ as in (1) and (2), this is the case if

$$
\begin{aligned}
h_{A B} & =2 \nabla_{(A} \xi_{B)}, \\
\omega & =0
\end{aligned}
$$


since $\bar{\gamma}$ and $W$ transform under the transformations (5) like tensors and scalars on $S_{c}$.

The vector field $P$, however, transforms under (5) according to $\varphi^{*} P=\left[P_{C}(\varphi(x)) \frac{\partial \varphi^{C}}{\partial x^{A}}+\bar{\gamma}_{A B} \frac{\partial \varphi^{-1 B}}{\partial x^{C}} \frac{\partial \varphi^{C}}{\partial U}\right] d x^{A}$. Thus, if $\xi$ is the induced vector field on $S_{c}$ of a 1-parameter group $\varphi_{\lambda}$ of diffeomorphisms,

$$
\left(\mathscr{L}_{\xi} P\right)_{A}=\xi^{K} \partial_{K} P_{A}+P_{C} \partial_{A} \xi^{C}+\gamma_{A C} \frac{\partial \xi^{C}}{\partial U} .
$$

Since $\boldsymbol{P}_{A}=0$ we find if

$$
P_{A}=\lambda Q_{A}
$$

(using (2.14) for $\gamma$ ) that

$$
Q_{A}=\xi_{A}^{\prime}-2 \mathscr{R}^{\prime} \xi_{A} .
$$

The problem is now to show that if $h_{A B}, \omega$ and $Q_{A}$ are such that $\left(\gamma_{A B}, W, \mathrm{P}_{A}\right)$ given by (1), (2), and (8) lies in $\mathscr{S}$, there exists a $\xi_{A}$ such that (6), (7), and (9) are satisfied. Clearly this requires showing that $\omega=0$ and, because of the uniqueness of the decomposition (3), that $\Phi_{A B}=0$. But if $\Phi_{A B}=0$ (6) has a solution $\xi_{A}^{*}\left(x^{B}, U\right)$, say, then all solutions of (6) are obtained by adding to $\xi_{A}^{*}$ arbitrary vector fields with vanishing Killing derivative. Thus it remains to solve

$$
\zeta_{A}^{\prime}-2 \mathscr{R}^{\prime} \zeta_{A}=Q_{A}-\xi_{A}^{\prime *}+2 \mathscr{R}^{\prime} \xi_{A}^{*}=\hat{Q}_{A}
$$

for $\zeta_{A}$ with $\bar{\nabla}_{(A} \zeta_{B)}=0$. A necessary condition for the existence of $\zeta_{A}$ is that (see (12))

$$
\hat{Q}_{A B}=\bar{\nabla}_{(A} \hat{Q}_{B)}=0 \text {. }
$$

It is also sufficient, since $\zeta_{A}\left(x^{B}, U\right)=r^{2}\left(\zeta_{A}\left(x^{B}\right)+\int_{U_{1}}^{U} \hat{Q}_{A} r^{-2} d U\right)$ for any $U_{1}$ and $\zeta_{1}\left(x^{B}\right)$ with $\bar{\nabla}_{(A} \zeta_{B)}=0$ clearly solves (10).

\section{b) Linearized Field Equations}

The field equations can now be linearized in a straightforward way.

(Observe that if all the indices of the first order quantities are raised and lowered by means of $\bar{\gamma}_{A B}$, we have in view of (2.14) and (3.3) for any tensor $K_{A B}:\left(K_{A}^{B}\right)^{\prime}=K_{A C}^{\prime} \bar{\gamma}^{C B}-2 \mathscr{R}^{\prime} K_{A}^{B}$. Moreover, since $\left(\bar{\Gamma}_{A B}^{C}\right)^{\prime}=0$,

$$
\left.\left(\nabla_{A} K_{B C}\right)^{\prime}=\bar{\nabla}_{A} K_{B C}^{\prime} \cdot\right)
$$

Then Eqs. (2.15), (2.16), (2.18) and (2.19) yield

$$
\begin{gathered}
2 \mathscr{R}^{\prime} \hat{\partial}_{A} \omega+\partial_{A} h^{\prime}-\bar{\nabla}_{B}\left(h_{A}^{B}\right)^{\prime}=2 \hat{\partial}_{A} Q_{B}^{B}-\bar{\nabla}_{B} Q_{A}^{B}, \\
4\left(r^{-2}+v \tilde{p}\right) \omega+2 W^{2} \mathscr{R}^{\prime} Q_{A}^{A}=\bar{\Delta} h-\bar{\nabla}^{A} \bar{\nabla}^{B} h_{A B}+r^{-2} h+W^{2} \mathscr{R}^{\prime} h^{\prime}, \\
\bar{\Delta} \omega-2 W^{2} \mathscr{R} \omega^{\prime}-4 v \tilde{p} \omega-\frac{1}{2} W^{2} h^{\prime \prime}-\frac{1}{2} M h^{\prime}+M Q_{A}^{A}+W^{2}\left(Q_{A}^{A}\right)^{\prime}=0,
\end{gathered}
$$


and

$$
2 \omega^{\prime}+4 M W^{-2} \omega+h^{\prime}-2 Q_{A}^{A}=0,
$$

respectively, where the quantities referring to the background metric are no longer in black type.

Substituting $h^{\prime}$ from (16) and $h^{\prime \prime}$ from its $U$-derivative into (15) leads to an equation for $\omega$ alone,

$$
\begin{aligned}
\bar{\Delta} \omega+W^{2} \omega^{\prime \prime}+(3 M- & \left.2 \mathscr{R}^{\prime} W^{2}\right) \omega^{\prime} \\
& +\left(2 M^{\prime}-2 M^{2} W^{-2}+8 M \mathscr{R}^{\prime}-4 v \tilde{p}\right) \omega=0 .
\end{aligned}
$$

To use the decomposition (3) for $h_{A B}$ in (13), (14) and (16) denote by $\bar{d}$ and $\bar{\delta}$ exterior differentiation and codifferentiation on differential forms in $S_{c}$. Then $\bar{\Delta}=-\bar{d} \bar{\delta}-\bar{\delta} \bar{d}$, which for 1 -forms $K=K_{A} d x^{A}$ is explicitly $\bar{\Delta} K=\left(\bar{\nabla}^{B} \bar{\nabla}_{B} K_{A}-\bar{R}_{A}^{B} K_{B}\right) d x^{A}=\left(\bar{\nabla}^{B} \bar{\nabla}_{B} K_{A}\right) d x^{A}-r^{-2} K$ (see, e.g. Goldberg [10]). If we define $K_{A_{B}}=\bar{\nabla}_{(A} \mathrm{K}_{B)}$, systematically for any 1 -form $K$, and let $\Phi=\Phi_{A}^{A}$ and $Q_{A}=Q_{A}+\xi_{A}^{\prime}-2 \mathscr{R}^{\prime} \xi_{A}$ it turns out that $\xi_{A}$ drops out of the Eqs. (13), (14) and (16); for we get successively from (13), (16), and (14)

$$
\begin{gathered}
\bar{d}\left(\Phi^{\prime}+2 \mathscr{R}^{\prime} \omega\right)=\bar{\delta} \bar{d} \hat{Q}-2 r^{-2} \hat{Q}, \\
2 \omega^{\prime}+4 M W^{-2} \omega+\Phi^{\prime}+2 \bar{\delta} \hat{Q}=0
\end{gathered}
$$

and

$$
\bar{\Delta} \Phi+r^{-2} \Phi=2 W^{2} \mathscr{R}^{\prime} \omega^{\prime}+4\left(M \mathscr{R}^{\prime}-r^{-2}-v \tilde{p}\right) \omega .
$$

\section{c) Solution for $\omega$}

Eq. (17) can be written in terms of the 3-dimensional Laplacian $\Delta$ which has in the spherically symmetric case the form

$$
\Delta f=W^{2} f^{\prime \prime}+M f^{\prime}+\bar{\Delta} f
$$

for any function $f$ on $\Sigma$. Since, if $g$ is any function of $U$,

$$
\Delta(g \omega)=g\left\{\bar{\Delta} \omega+W^{2} \omega^{\prime \prime}+\left[2 W^{2}(\ln g)^{\prime}+M\right] \omega^{\prime}+\left[M(\ln g)^{\prime}+W^{2} g^{\prime \prime} g^{-1}\right] \omega\right\}
$$

we can choose $g$ such that $2 W^{2}(\ln g)^{\prime}+M=3 M-2 \mathscr{R}^{\prime} W^{2}$ whence up to a constant, $g=m / r$. Then, after some calculations, (17) is seen to be equivalent to

$$
\Delta \tilde{\omega}=F \tilde{\omega}
$$

where $\tilde{\omega}=m r^{-1} \omega$ and

$$
F(U)=-M^{\prime}+2 M^{2} W^{-2}-6 M \mathscr{R}^{\prime}+3 v \tilde{p}+v W^{2} .
$$

If now the deformed solution is to lie in $\mathscr{P}$ we must have $W=\boldsymbol{W}(1+\lambda \omega)$ $=m r^{-2}+0\left(r^{-3}\right)$ for $r \rightarrow \infty$ with, possibly, a different $m$ and $W^{2}=\left(\partial_{i} \partial_{j} W^{2}\right)_{0} y^{i} y^{j}+0\left(|y|^{3}\right)$ in terms of a normal coordinate system near the center. 
It follows by arguments similar to those in the last chapter that

$$
\tilde{\omega}=0\left(z^{2}\right) \text { near the center }
$$

and $\tilde{\omega}=0\left(r^{-1}\right)$ or $0\left(\mathrm{r}^{-2}\right)$ for $r \rightarrow \infty$. In particular, $\tilde{\omega}$ is regular everywhere on $\Sigma$.

If now $F(U)$ in (21) were everywhere positive on $\Sigma$ it would follow immediately that $\tilde{\omega}$ vanishes. This is not quite true, however, due to the (only negative) term $-6 M \mathscr{R}^{\prime}$. (Near the center $F=-\frac{1}{5}\left(3 v \tilde{p}_{0}-M_{0}^{\prime}\right)+0(z)$, which is manifestly negative.) A closer analysis is therefore needed.

As a regular function on each $S_{c} \tilde{\omega}$ can be expanded in terms of spherical harmonics. Let

$$
\tilde{\omega}=\sum_{l \geqq 0} K_{l}(U) Y_{l}(\theta, \varphi)
$$

where $Y_{l}$ is any (normalized) linear combination of spherical harmonics of order $l$, i.e. satisfies $\Lambda Y_{l}+l(l+1) Y_{l}=0$ and $\int_{S^{2}} Y_{l}^{2} d \Omega=1$, where $\Lambda$ is the Laplacian restricted to the unit sphere.

Then $\bar{\Delta}=r^{-2} \Lambda$ and (4.21) reduces to an equation for $K_{l}$,

$$
W^{2} r^{2} K_{l}^{\prime \prime}+M r^{2} K_{l}^{\prime}=\left[r^{2} F+l(l+1)\right] K_{l}, \quad l=0,1,2, \ldots
$$

We first investigate this equation near the center where according to (23) $K_{l}$ must vanish for all $l$. Since $\tilde{\omega}$ is regular we can expand in terms of at least a few powers of $z$.

Comparing the first coefficients of the powers of $z$ in (25) shows that $K_{l}(z)=a_{0 l} z^{l}+0\left(z^{l+1}\right)$ near the center. In view of (23) and (24) we see that $K_{0}=K_{1}=0$.

It will now be shown that the remaining multipoles in the solution for $\tilde{\omega}$ also vanish because the function

$$
\tilde{F}_{l}=F+\frac{l(l+1)}{r^{2}}>0 \text { for all } l \geqq 2
$$

everywhere on $\Sigma$. Indeed, if (26) holds (25) or $\Delta K_{l}=W^{2} K_{l}^{\prime \prime}+M K_{l}^{\prime}=\tilde{F}_{l} K_{l}$ implies $\Delta K_{l}^{2}=2 K_{l} \Delta K_{l}+2 W^{2} K_{l}^{\prime 2}=2 F_{l} K_{l}^{2}+2 W^{2} K_{l}^{\prime 2} \geqq 0$ so that $K_{l}^{2}$ can not have a maximum on $\Sigma$. Since $\lim _{r \rightarrow \infty} K_{l}=0$ this implies that $K_{l}=0$ on all of $\Sigma$.

There remains the proof of (26). Because $F$ is finite at the center and equal to $v W^{2}$ in the exterior vacuum region $F_{l}$ is strictly positive near the the center and in vacuum for all $l \geqq 1$. To estimate $F_{l}$ in the region between we find from (22) with (2.10) $F \geqq 2 M^{2} W^{-2}-6 M \mathscr{R}^{\prime}+v\left(5 M+W^{2}\right)$. In the Newtonian case $(v=0)$ it is easy to conclude that this is $\geqq-6 r^{-2}$. For, from (3.5) and (3.6), $\mathscr{R}{ }^{\prime}=r m^{-1}$, such that $F \geqq 2 M^{2} m^{-2} r^{4}-6 M^{-1} r$. At any fixed point outside the center $r m^{-1}$ has some fixed positive value 
and $F$ regarded as a quadratic function of $M$ is immediately seen to be $\geqq-\frac{9}{2} r^{-2}$. The estimate in the relativistic case is somewhat more complicated. It is given in an appendix under the assumption that $0 \leqq 3 p \leqq \varrho$.

\section{d) Solution for $\Phi_{A B}$ and $Q_{A}$}

With $\omega=0$ Eq. (20) becomes $\bar{\Delta} \Phi+r^{-2} \Phi=0$ or, restricted to any sphere $S_{c}, A \Phi+\Phi=0$. Since there are no regular solutions on the sphere of this equation it follows that $\Phi=0$. Then, since also $\bar{\nabla}^{A} \Phi_{A B}=0$, Lemma 1 implies $\Phi_{A B}=0$. Now (18) reads $\bar{\delta} \bar{d} \hat{Q}-2 r^{-2} \hat{Q}=0$ and from (19) we have

$$
\bar{\delta} \hat{Q}=0
$$

whence $\bar{\Delta} \hat{Q}+2 r^{-2} \hat{Q}=0$, or, in terms of coordinates, $\bar{\nabla}^{C} \bar{\nabla}_{C} \hat{Q}_{A}+r^{-2} \hat{Q}_{A}=0$. Differentiation and use of (27) and the Ricci identity give $\nabla^{C} \nabla_{C} Q_{A B}$ $=r^{-2} Q_{A B}$ and therefore $Q_{A B}=0$ by the same argument as was used for $K_{l}$. This completes the proof of

Theorem 3. A static spherically symmetric asymptotically Euclidean space-time representing vacuum and a perfect fluid with $0 \leqq 3 v p \leqq \varrho$ and an equation of state $\varrho(p)$ satisfying $0<\frac{d p}{d \varrho} \leqq \infty$ in a compact domain does not admit any (non-trivial) static deformations with the same equation of state and the same maximal (i.e. central) pressure and the same minimal value of the gravitational potential.

The author is grateful to Professors A. Avez and A. Taub for several helpful discussions.

\section{Appendix}

A proof that (4.26) holds in the general relativistic case can be given by means of the following elementary estimates.

Using (3.6) and (3.5) and the new variables

$$
u=\frac{m}{r}, \quad v=\tilde{p} r^{2}, \quad x=\frac{1}{3} M r^{2} u^{-1},
$$

(Note that they are all regular functions with values $0,0,1$, respectively, at the center.) We write (4.26) in the form

$$
F r^{2} \geqq 18 x^{2}+15 u x+u^{2}-18 x \sqrt{1+u^{2}+v} \geqq-6 .
$$

In view of the conditions $0 \leqq 3 p \leqq \varrho$ or equivalently $0 \leqq v \leqq x u$ it will be sufficient to show that

$$
18 x^{2}+15 x u+u^{2}+6 \geqq 18 x \sqrt{1+u+u x}
$$


for all positive values of $u$ and $x$. This is equivalent to

$$
\begin{aligned}
f=324 x^{4}+216 u x^{3}-63 u^{2} x^{2}-108 x^{2} & \\
& +10 u^{3}+60 u x+u^{4}+12 u+36 \geqq 0 .
\end{aligned}
$$

For $u=0$ we have $f=(18 x-3)^{2}+27>0$. Then letting $x=\alpha u$ for $0 \leqq u<\infty$ and $0 \leqq \alpha<\infty$ gives

$$
f=A u^{4}+12 B u^{2}+36
$$

with $A=324 \alpha^{4}+216 \alpha^{3}-63 \alpha^{2}+10 \alpha+1$ and $B=-9 \alpha^{2}+5 \alpha+1$. It will be enough to show that $f$ in (1) is positive for all $u$ and all $\alpha \geqq 0$. This is the case iff (a) $A \geqq 0$ and (b) $B>0$ or $A \geqq B^{2}$. But this condition does hold as is easily seen by a closer inspection of the two functions $A(\alpha)$ and $B(\alpha)$ for $\alpha \geqq 0$.

\section{References}

1. Avez, A.: Ann. Inst. Henri Poincaré 1, 291-300 (1964).

2. - C. R. Acad. Sci. Paris 257, 3316-3318 (1963).

3. Berger, M., Ebin, D.: J. Diff. Geom. 3, 379-392 (1969).

4. Bondi, H.: Proc. Roy. Soc. A 282, 303-317 (1964).

5. Carleman, T.: Math. Z. 3, 1-7 (1919).

6. Deser, S.: Ann. Inst. Henri Poincaré 7, $149-188$ (1967).

7. - Ann. Inst. Henri Poincaré 8, 269-273 (1968).

8. Ehlers, J., Kundt, W.: Artıcle in L. Witten (ed.): Gravitation, an introduction to current research. New York: Wiley 1962.

9. Eisenhardt, L. P.: Riemannian geometry. Princeton: Princeton University Press 1950

10. Goldberg, S. I.: Curvature and homology. New York: Academic Press 1962.

11. Künzle, H. P.: Proc. Roy. Soc. A 297, 244-268 (1967).

12. Landau, L. D., Lifshitz, E. M.: The classical theory of fields. Oxford: Pergamon Press 1962.

13. Lichnerowicz, A.: Théories relativistes de la gravitation et de l'éléctromagnetisme. Paris: Masson 1955.

14. Lıchtenstein, L.: Gleichgewichtsfiguren rotierender Flüssigkeiten. Berlin-GöttingenHeidelberg: Springer 1933.

15. Milnor, J.: Morse theory. Princeton: Princeton University Press 1963.

16. Morse, M.: Trans. Am. Math. Soc. 27, 345-396 (1925).

17. Sachs, R. K.: Article in B. Hoffmann (ed.): Perspectives in geometry and relativity. Bloomington: Indiana University Press 1966.

H. P. Künzle

Department of Mathematics

University of Alberta

Edmonton 7, Alberta, Canada 